\title{
Effects of Naringenin on Oxidative Damage and Cell Apoptosis in Diabetic Retinopathy Rats via the Nrf2/ARE Signaling Pathway
}

\begin{abstract}
YUANMING WANG, JIAO YUE ${ }^{1}$, CHUN CHEN² AND YINGJUAN HAO ${ }^{3 *}$
Department of Endocrinology, Affiliated Hospital of Gansu University of Traditional Chinese Medicine, Lanzhou 730020, Gansu Province, ${ }^{1}$ Chengdu Center for Disease Control and Prevention, China Railway Chengdu Bureau Group Co., Ltd., Chengdu 610031, Sichuan Province, '2Lanzhou Chengguan District Center for Disease Control and Prevention, Lanzhou 730000, Gansu Province, ${ }^{3}$ Department of Ophthalmology, Nanjing Tongren Hospital, School of Medicine, Southeast University, Nanjing 210000, Jiangsu Province, China
\end{abstract}

Wang et al.: Effects of Naringenin on Diabetic Retinopathy

\begin{abstract}
We aimed to assess the effects of naringenin on oxidative damage and cell apoptosis in diabetic retinopathy rats via the nuclear factor erythroid 2-related factor 2/antioxidant response element signaling pathway. 70 rats were randomly divided into normal and model groups. Diabetic model was established through continuous intraperitoneal injection of streptozotocin. Model rats were further randomly divided into model, low dose and high dose groups. Body weight and blood glucose level changes were observed. Retinal thickness was detected through optical coherence tomography. The content of malondialdehyde, activities of superoxide dismutase and glutathione peroxidase and number of reactive oxygen species-positive cells in retinal tissues were determined. Cell apoptosis was detected by terminal deoxynucleotidyl transferasemediated dUTP nick end labeling staining. The protein expressions of nuclear factor erythroid 2-related factor 2, NAD(P)H quinone oxidoreductase 1 and heme oxygenase-1 were detected by western blotting. Compared with model group, body weight rose; while blood glucose level and retinal thickness declined in low and high dose groups. The number of reactive oxygen species-positive cells decreased in high dose group compared with that in model group. Malondialdehyde content and apoptotic index increased, while superoxide dismutase and glutathione peroxidase activities decreased in model group compared with those in normal group. Nuclear factor erythroid 2-related factor 2, heme oxygenase-1 and NAD(P)H quinone oxidoreductase 1 protein expressions were higher in low and high dose groups than those in model group. Naringenin protects against retinal damage in diabetic rats probably by resisting oxidative damage and inhibiting retinal tissue apoptosis through regulating protein expressions of the nuclear factor erythroid 2-related factor 2/antioxidant response element signaling pathway.
\end{abstract}

Key words: Cell apoptosis, diabetic retinopathy, naringenin, oxidative damage

Diabetes is a chronic metabolic disease characterized by high blood glucose level, which can lead to chronic injury and dysfunction of various organs and tissues, such as the eye, heart and nervous system ${ }^{[1]}$. Chronic complications caused by diabetes, especially diabetic microangiopathy, are the main causes of the sharp decline in the quality of life of diabetic patients. A recent study has shown that Diabetic Retinopathy (DR) is one of the most common microvascular complications of diabetes and also the leading cause of blindness among working-age population around the world ${ }^{[2]}$. According to statistics, about $30 \%$ of diabetic patients are accompanied by varying degrees of DR. At present, the pathogenesis of the disease remains unclear. It has been found that oxidative stress

*Address for correspondence E-mail: kingdomchuaner@163.com

September-October 2021 may be one of the pathogeneses of DR. Clinically, the onset of DR is characterized by the increase in vascular permeability, occlusion and formation of non-perfusion area and its significant signs are the destruction of blood retinal barrier and increase in exudation or the pathological hyperplasia of retinal vessels. If there are no effective improvement measures, new vessels may break through the inner limiting membrane and enter

This is an open access article distributed under the terms of the Creative
Commons Attribution-NonCommercial-ShareAlike 3.0 License, which
allows others to remix, tweak, and build upon the work non-commercially,
as long as the author is credited and the new creations are licensed under
the identical terms Accepted 04 September 2021 Revised 03 June 2021 Received 31 January 2020 Indian J Pharm Sci 2021;83(5):899-905 
the vitreous cavity, resulting in vitreous hemorrhage and retinal detachment ${ }^{[3]}$. Naringenin (NAR) is a natural flavonoid compound and it has been confirmed to be able to regulate metabolism and resist inflammation, oxidation and fibrosis, with broad application prospects in the fields of medicine, health care and food ${ }^{[4]}$. NAR can increase the expressions of brain-derived neurotrophic factors, tropomyosin-related kinase B and synaptophysin in the retina of DR rats and improve the levels of apoptosis-modulating proteins, thereby exerting a certain protective effect against retinal nerve damage. The Nuclear factor erythroid 2-related factor 2 (Nrf2)/Antioxidant Response Element (ARE) signaling pathway is the most important endogenous antioxidant pathway so far, whose role in regulating oxidative stress has been paid increasing attention to ${ }^{[5,6]}$. NAR may reduce oxidative stress damage in DR rats through activating the Nrf2/ARE signaling pathway ${ }^{[7]}$. In the present study, to explore the effect of NAR on DR, the rat model of DR was established to study the effects of NAR on oxidative damage and cell apoptosis in DR rats, the protective effect of NAR on the retina was observed through regulating the Nrf2/ARE signaling pathway and the mechanism of NAR was also investigated, hoping to provide more experimental bases for the treatment of DR with NAR and the clinical medication of DR.

\section{MATERIALS AND METHODS}

\section{Experimental animals, reagents and apparatus:}

Male rats ( $2 \mathrm{w}$ old, $120.0 \pm 1.0 \mathrm{~g})$ were purchased from Beijing Vital River Laboratory Animal Co., Ltd., and fed in the laboratory animal center of our institute. NAR standards (purity: $98 \%$ ) and Terminal Deoxynucleotidyl Transferase-mediated dUTP Nick End Labeling (TUNEL) assay reagent were provided by Sigma (USA), anesthetics were purchased from Thermo Fisher $\left(\mathrm{Gibco}^{\mathrm{TM}}\right)$ and western blotting kits were sourced from Rebstock (Germany).

Bio-Rad gel imaging system was bought from Bio$\mathrm{Rad}, \mathrm{a}-80^{\circ}$ cryogenic refrigerator was purchased from WIGGENS (Germany), a fluorescence microscope was bought from Thermo Fisher (USA), a confocal microscope was sourced from OLYMPUS (Japan) and a high-speed low-temperature centrifuge was provided by Beijing Liuyi Instrument Factory.

\section{Modeling and grouping:}

After adaptive feeding, the rats were randomly divided into normal group $(n=10)$, model group $(n=20)$, low dose group $(n=20)$ and high dose group $(n=20)$. The diabetic rat model was established through intraperitoneal injection of streptozotocin ${ }^{[8]}$ except normal group (fed with citrate buffer solution). Specifically, $1 \%$ streptozotocin was gavaged at $80 \mathrm{mg} /(\mathrm{kg} / \mathrm{d})$ regularly in the morning every day for $3 \mathrm{~d}$. The blood glucose level in the caudal vein $>16.7 \mathrm{mmol} / 1$ indicated successful modeling and such rats were enrolled into model group and drug administration groups for experiments. The rats in low dose group (15 mg/kg NAR) and high dose group (95 mg/kg NAR) were gavaged with an equal volume of NAR at different concentrations, while those in normal group and model group were given the same volume of citrate buffer solution. The efficacy was detected in each group for $12 \mathrm{w}$.

\section{Detection of observation indices:}

During treatment, the conditions of rats were observed in each period. The body weight of rats was recorded at 10:00 am on Monday for every $5 \mathrm{w}$. After abdominal anesthesia, the blood was drawn from the caudal vein for every $5 \mathrm{w}$ and the blood glucose level was measured by glucose oxidase method.

\section{Optical Coherence Tomography (OCT):}

The retinal thickness was detected in each group through OCT using Micron IV retinal imaging system. Specifically, the rats were anesthetized and the pupils were dilated with $10 \mathrm{~g} / 1$ tropicamide eye drops. The cornea was lubricated with GenTeal gel and the Micron eyepieces were placed where the gel was in direct contact with the eyes. OCT images were captured through full scan and 3 images were taken for each eye in the directions above and below the optic nerve, nose and tempus.

\section{Detection of oxidative stress indices:}

After drug administration, the body weight was measured and the rats were anesthetized through intraperitoneal injection of pentobarbital sodium $(60 \mathrm{mg} / \mathrm{kg})$. Next, the eyeballs were removed and the retina was peeled off under an ophthalmic operating microscope. One portion of the retinal tissues was prepared into homogenate and centrifuged and the supernatant was harvested. Then the activity of Superoxide dismutase (SOD) and Glutathione peroxidase (GSH-Px) and the content of Malondialdehyde (MDA) were detected using xanthine oxidase method, colorimetry and thiobarbituric acid method, respectively. The other portion of the retinal tissues was stored in $40 \mathrm{~g} / \mathrm{l}$ formaldehyde solution for later use. 


\section{Detection of cell apoptosis by TUNEL assay:}

One portion of the retinal tissues was washed and fixed with $4 \%$ paraformaldehyde, embedded in paraffin and sliced into sections. The sections were eluted with xylene and gradient ethanol, rinsed with phosphate buffer solution, incubated in ultrapure water containing $2 \%$ protein kinase $\mathrm{K}$ at room temperature for $15 \mathrm{~min}$ and washed with ultrapure water twice. Then TUNEL solution was added for incubation at $37^{\circ}$ for $1 \mathrm{~h}$ in the dark and the sections were washed with phosphate buffer solution twice and sealed with 4',6-diamidino2-phenylindole and $20 \%$ glycerol buffer. Finally, the sections were observed under a high-power optical microscope and the images were acquired and analyzed.

Measurement of mitochondrial membrane potential:

The cells were inoculated in the confocal dish (10 $\times 10^{3}$ cells/well). After adherence, the cells were treated with drugs and the medium was discarded. Then the cells were washed with phosphate buffer solution, mixed evenly with staining working solution and incubated in the incubator at $37^{\circ}$ for $20 \mathrm{~min}$. After that, the supernatant was aspirated and washed twice with staining buffer. After $1 \mathrm{ml}$ of medium was added, the red and green color changes in the mitochondrial membrane potential in each group were observed under the confocal microscope. The cells were counted in 10 randomly selected visual fields and the apoptotic index was calculated and the average was taken in each group. Apoptotic index $(\%)=$ number of apoptotic cells/ total number of cells $\times 100$.

\section{Detection of retinal tissue protein expressions:}

After the medium was discarded, the cells in each group were digested with trypsin, centrifuged and collected. The protein lysis buffer was added and mixed evenly in each group, followed by lysis on ice for $30 \mathrm{~min}$. Then the lysate was centrifuged in a table-type highspeed refrigerated centrifuge and the supernatant was collected, from which the total protein was extracted and quantified using bicinchoninic acid (BCA) method according to the instructions. Next, the Sodium dodecyl sulphate-Polyacrylamide Gel Electrophoresis (SDSPAGE) gel was prepared and the protein was subjected to electrophoresis, transferred onto a membrane, mounted and incubated with primary and secondary antibodies, followed by exposure and scanning analysis using image analysis software. With Glyceraldehyde-3-phosphate dehydrogenase (GAPDH) as an internal reference, the protein expressions of Nrf2, Heme Oxygenase-1
(HO-1) and $\mathrm{NAD}(\mathrm{P}) \mathrm{H}$ quinone oxidoreductase 1 (NQO1) were determined.

\section{Statistical analysis:}

Statistical Package for the Social Sciences (SPSS) 20.0 software was used for statistical analysis and Graphpad Prism 5.0 was used for plotting. Intergroup comparison was conducted using t test. $\mathrm{p}<0.05$ was considered to be statistically significant.

\section{RESULTS AND DISCUSSION}

The body weight of rats rose perpendicularly within $12 \mathrm{w}$ in normal group. Compared with normal group, model group had significantly decreased body weight from the $4^{\text {th }} \mathrm{W}$ to the $12^{\text {th }} \mathrm{W}$ and the difference was statistically significant $(p<0.05)$. Compared with model group, low dose group and high dose group had significantly increased body weight from the $8^{\text {th }} \mathrm{W}$ to the $12^{\text {th }} \mathrm{w}(\mathrm{p}<0.05)$ and the body weight was greater in high dose group, with a statistically significant difference $(p<0.01)$. Besides, the blood glucose level had no obvious changes within $12 \mathrm{w}$ in normal group, while it rose significantly at 4,8 and $12 \mathrm{w}$ in model group compared with that in normal group $(\mathrm{p}<0.05)$. Moreover, it declined significantly at 4, 8 and $12 \mathrm{w}$ in low dose group compared with that in model group $(\mathrm{p}<0.05)$, while it was lower at 4 and $8 \mathrm{w}(\mathrm{p}<0.05)$ but higher at $12 \mathrm{w}$ in high dose group than that in model group, with statistically significant differences $(p<0.01)$ (fig. 1).

It was observed through OCT that the retinal thickness was comparable in each group at $0 \mathrm{w}$ after modeling and it was significantly increased within $4-12 \mathrm{w}$ in model group. The retinal thickness was significantly smaller at 8 and $12 \mathrm{w}$ in low dose group than that in model group $(\mathrm{p}<0.05)$, while it was smaller at 4 and $8 \mathrm{w}$ $(\mathrm{p}<0.05)$ and significantly smaller at $12 \mathrm{w}$ in high dose group than that in model group, showing statistically significant differences ( $<<0.01)$ (fig. 2).

The level of MDA was significantly higher, while the levels of SOD and GSH-Px were significantly lower in model group than those in normal group $(\mathrm{p}<0.05)$. Compared with those in model group, the level of MDA significantly declined $(\mathrm{p}<0.05)$, while the level of GSH-Px significantly rose in low dose group $(\mathrm{p}<0.05)$ and they were improved more significantly in high dose group (p<0.01) (fig. 3).

The fluorescent probe binding to intracellular ROS will produce high intensity green fluorescence in cells and the brightness and quantity of the green fluorescence 

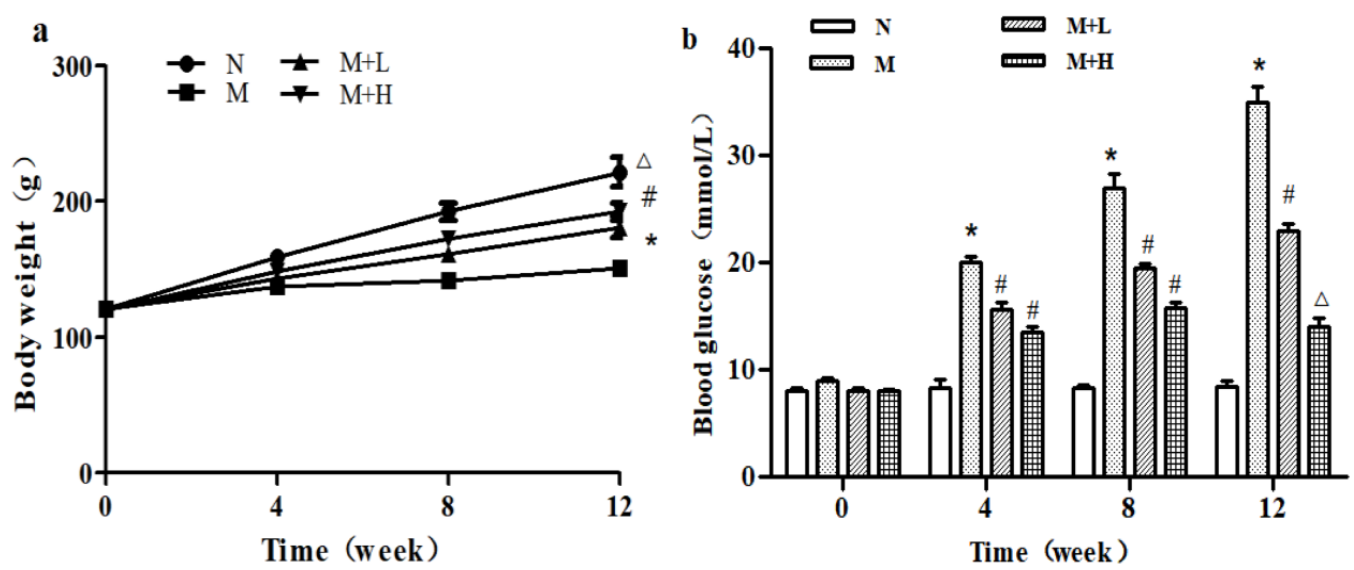

Fig. 1: Observation results of rats in each group after treatment, (N) Normal group; (M) model group; (M+L) low dose group; $(\mathrm{M}+\mathrm{H})$ high dose group

Note: * $\mathbf{p}<0.05$ vs. normal group, ${ }^{\#} p<0.05$ and ${ }^{\Delta} \mathbf{p}<0.01$ vs. model group

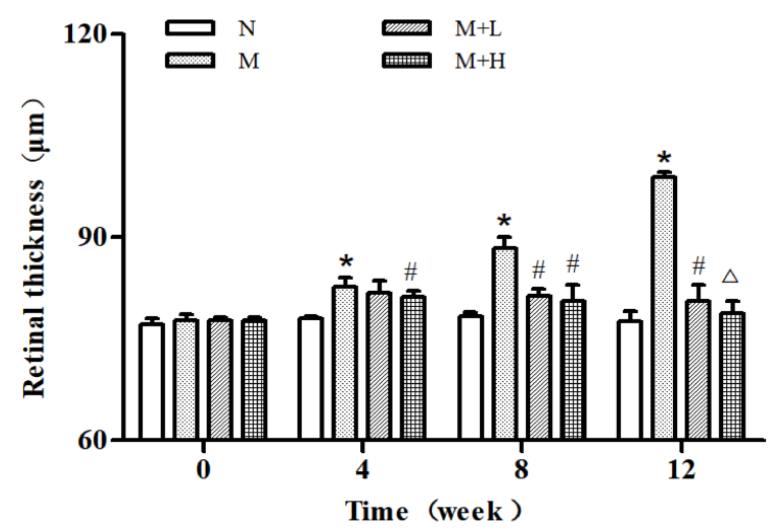

Fig. 2: Retinal thickness of rats in each group after treatment, (N) normal group; (M) model group; (M+L) low dose group; (M+H) high dose group

Note: ${ }^{*} p<0.05$ vs. normal group, ${ }^{\#} p<0.05$ and ${ }^{\triangle} p<0.01$ vs. model group
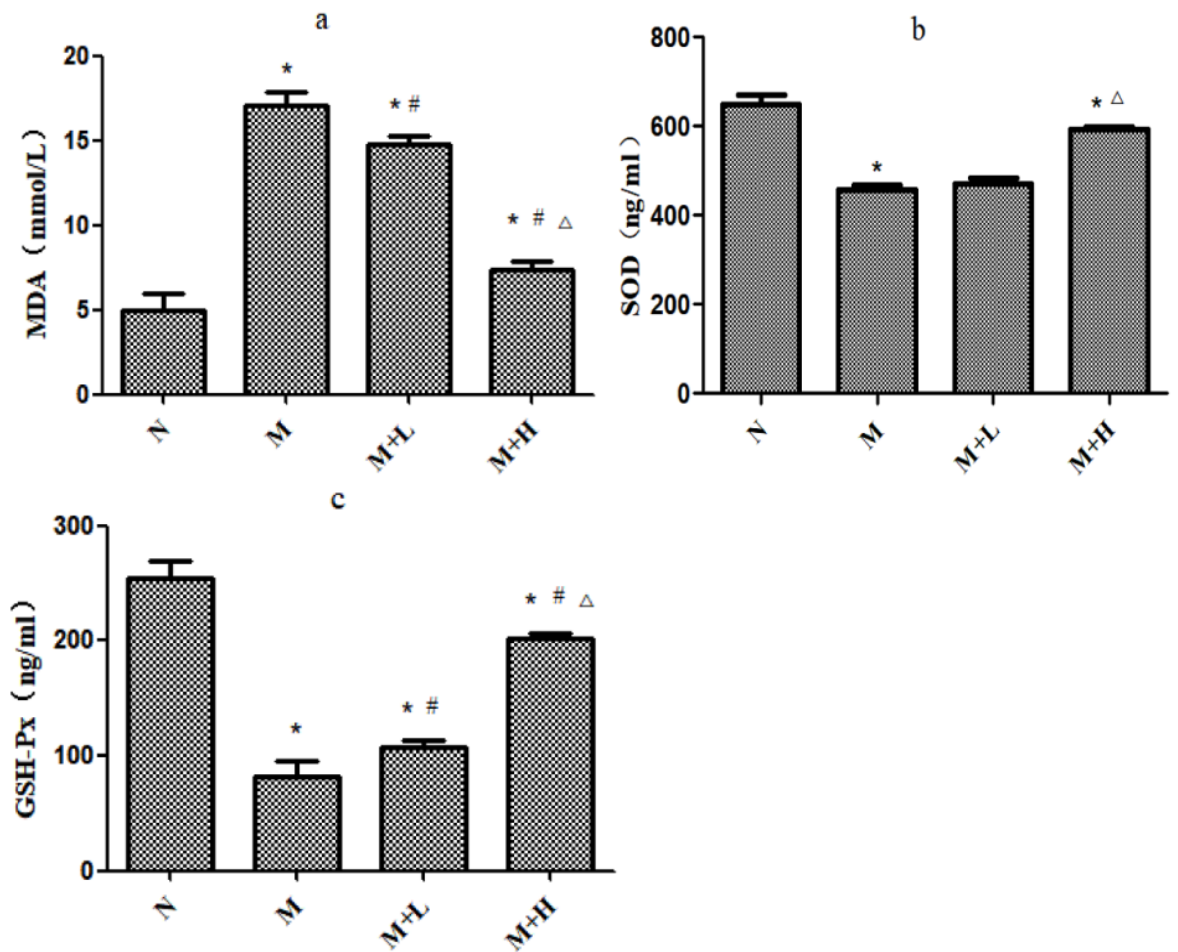

Fig. 3: Levels of MDA, SOD and GSH-Px in retinal tissues in each group after treatment, (N) normal group; (M) model group; $(\mathrm{M}+\mathrm{L})$ low dose group; $(\mathrm{M}+\mathrm{H})$ high dose group

Note: * $\mathbf{p}<0.05$ vs. normal group, ${ }^{\#} \mathbf{p}<0.05$ and ${ }^{\triangle} \mathbf{p}<0.01$ vs. model group 
reflect the amount of ROS in each group. It was found that the number of ROS-positive cells was increased in model group compared with that in normal group $(p<0.05)$, while it was decreased in low dose group $(p<0.05)$ and significantly declined in high dose group in contrast with that in model group $(p<0.01)$. It can be seen that NAR can reduce the accumulation of ROS (fig. 4).

In TUNEL staining, the brown yellow staining indicates positive expression and the normal cell nucleus is stained blue with hematoxylin. In this study, the results of TUNEL staining revealed that the apoptosis was not obvious in normal group, while a large number of positive apoptotic cells could be seen under the microscope in model group, showing a statistically significant difference between the two groups $(\mathrm{p}<0.05)$. Compared with that in model group, the positive expression significantly declined in low dose group and high dose group and the apoptosis was also relieved with the increase of dose of NAR $(\mathrm{p}<0.01)$ (fig. 5).

The protein expressions of Nrf2, HO-1 and NQO1 in retinal tissues were remarkably lower in model group than those in normal group $(\mathrm{p}<0.05)$. Besides, they were remarkably higher in low dose group and high dose group than those in model group in an NAR concentration dependent manner and they were increased more significantly in high dose group $(\mathrm{p}<0.01)$ (fig. 6).

DR is the most important complication in the eyes of diabetic patients and also one of the common microangiopathy in diabetic patients, mainly manifested as apoptosis of retinal ganglion cells, neuroinflammatory injury in vessels, blood-retinal barrier breakdown and abnormalities in function and structure of multiple cells, which can lead to blindness in severe cases, bringing

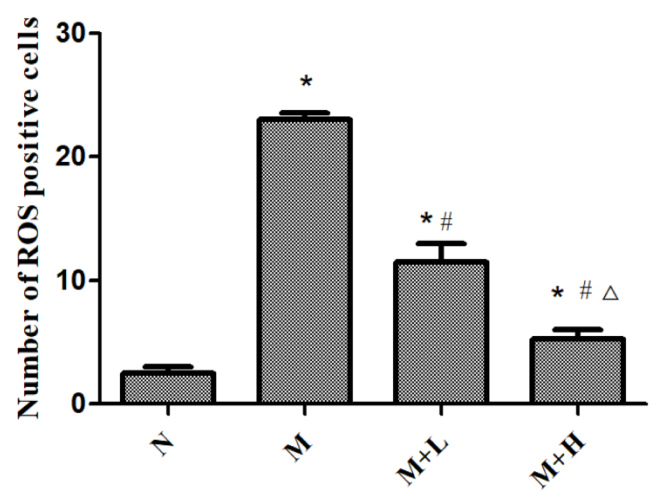

Fig. 4: ROS-positive cells under the confocal microscope in each group after treatment, $(\mathrm{A} / \mathrm{N})$ normal group; $(\mathrm{B} / \mathrm{M})$ model group; $(\mathrm{C} / \mathrm{M}+\mathrm{L})$ low dose group; $(\mathrm{D} / \mathrm{M}+\mathrm{H})$ high dose group Note: *p $<0.05$ vs. normal group, ${ }^{\#} p<0.05$ and ${ }^{\triangle} p<0.01$ vs. model group

September-October 2021 about heavy burdens to the patients, their families and society ${ }^{[9]}$. The treatment methods of DR are only suitable for patients at the middle or advanced stages and it cannot reverse the visual impairment in patients despite its ability to block the continuous development of the disease. Therefore, early intervention and treatment of DR patients have important clinical significance. Establishing animal models of diabetes and DR is the basis for the research on the pathogenesis of DR, which is of important significance. About 100 y ago, animal models of diabetes were already successfully established by humans and became an important tool for studying diabetes and its complications. It is of profound significance to explore the pathogenesis of DR using animal models and alleviate the severity of DR for patients with DR.

In recent years, the research on extracts of biologically active substances has become more and more

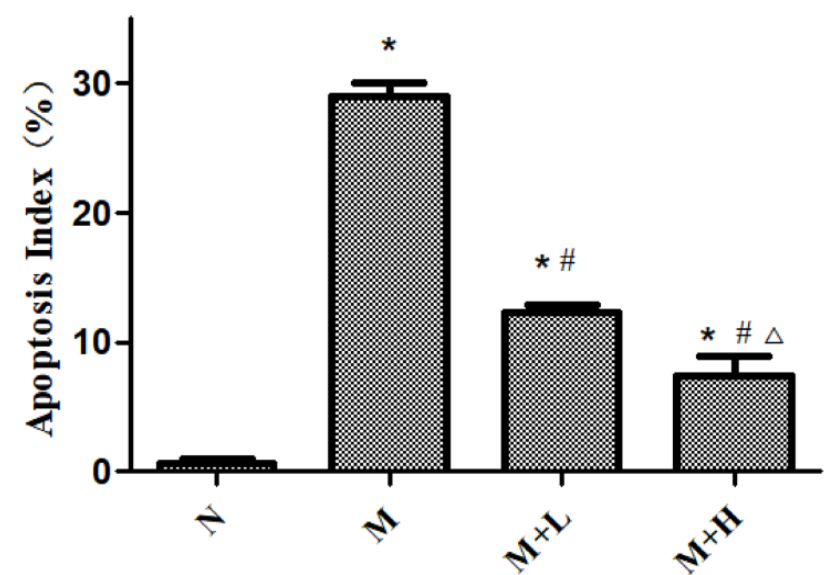

Fig. 5: TUNEL staining results of retinal tissues in each group, (A/N) normal group; (B/M) model group; $(\mathrm{C} / \mathrm{M}+\mathrm{L})$ low dose group; $(\mathrm{D} / \mathrm{M}+\mathrm{H})$ high dose group

Note: * $p<0.05$ vs. normal group, ${ }^{*} p<0.05$ and ${ }^{\Delta} \mathbf{p}<0.01$ vs. model group

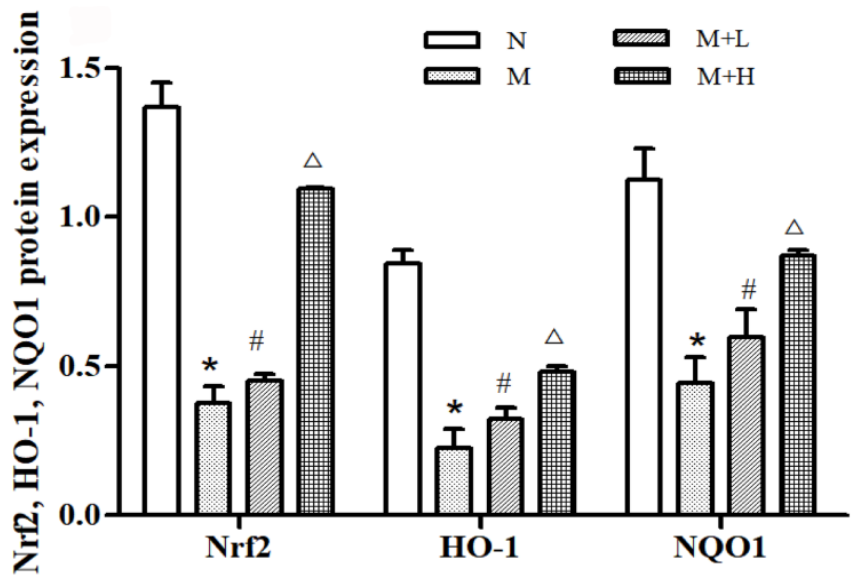

Fig. 6: Nrf2, HO-1 and NQO1 protein expressions in retinal tissues detected by Western blotting. (N) Normal group; (M) model group; $(\mathrm{M}+\mathrm{L})$ low dose group; $(\mathrm{M}+\mathrm{H})$ high dose group Note: ${ }^{*} p<0.05$ vs. normal group, ${ }^{*} p<0.05$ and ${ }^{\Delta} p<0.01$ vs. model group 
popular and substances extracted from some plants have a protective effect against damage caused by hyperglycemia in human body. For example, puerarin is related to reducing the inflammatory response and oxidative stress damage of retinal tissues and it has been found that the rats in model group have significantly lower body mass and evidently higher blood glucose level, infiltrating amount of Evans blue in retinal tissues and apoptotic index of ganglion cells than normal control group. Erianin possesses a protective effect against oxidative damage and apoptosis of the retina upon diabetes mellitus and the improvement effect is positively correlated with its dose $\mathrm{e}^{[10]}$, but its mechanism is still under study. NAR is a dihydroflavonoid compound mainly present in rutaceous plants, such as grapefruit, grapes, citrus fruits and tomatoes, which possesses a variety of biological activities, such as anti-oxidation, anti-tumor, anti-inflammation and anti-virus. For example, NAR has a protective effect against cardiac injury caused by ischemia-reperfusion and it can alleviate peripheral neuropathic pain induced by paclitaxel in mice ${ }^{[11]}$. Priscilla et al. ${ }^{[4]}$ found through in vivo and in vitro experiments that NAR could suppress the activity of alpha $(\alpha)$-glucosidase in diabetic rats, delay the absorption of glucose in the intestine after meals and lower the postprandial blood glucose level, indicating that it has broad clinical application prospects. In this study, the rat model of DR was successfully established and NAR was used as an effective drug for DR. It was found that the body weight of rats in model group was significantly smaller than that in normal group. After NAR treatment for 12 consecutive $\mathrm{w}$, the blood glucose level of DR rats prominently declined. It was observed through OCT that the retinal thickness of rats was greatly increased in low dose group and high dose group compared with that in model group. Compared with model group, low dose group and high dose group had an obviously decreased level of MDA and obviously enhanced activity of SOD and GSH-Px. Moreover, it was observed under the optical microscope that oxidative stress damage was caused to retinal ganglion cells and the apoptotic index was higher in DR rats in model group. Furthermore, it was found under the fluorescence microscope that the level of ROS rose in model group compared with that in normal group, while it markedly declined after NAR treatment, indicating that NAR may reduce cell apoptosis caused by oxidative stress through suppressing the accumulation of intracellular ROS. The detection results of mitochondrial membrane potential in each group further proved that the number of apoptotic cells declined in high dose group.

Nrf2 is a key transcription factor maintaining oxidative stress in cells. Under normal conditions, Nrf2 is coupled to cytoskeleton related proteins and binds to cytoplasmic actin. It is believed that the core signaling pathway of $\mathrm{Nrf2}$ is an important antioxidant pathway, which plays a vital role in keeping the intracellular redox homeostasis. A large number of downstream molecules regulated by Nrf2 are able to resist oxidative stress and apoptosis, regulate inflammatory injury and relieve cytotoxicity ${ }^{[12]}$. ARE is a specific promoter deoxyribonucleic acid (DNA)-binding sequence, which regulates the transcription of oxidoreductases, phase II metabolic enzymes and transporter genes, thereby enhancing the anti-oxidative stress and damage repair ability of cells. Nrf2, HO-1 and NQO1 pathways are all important anti-oxidative stress pathways. Huang et al. ${ }^{[13]}$ found through comparison that the protein expressions of Nrf2 and HO-1 rose in artemisinin pre-protection group, indicating that artemisinin can activate $\mathrm{Nrf2}$ by regulating the Nrf2 and HO-1 pathways, thereby improving the anti-oxidative stress ability of cells. Grape seed proanthocyanidin is a flavonoid compound and it is able to raise the expression levels of Nrf2 and HO-1 in the retina of DR rats and improve the oxidative stress-mediated retinal damage in DR rats through activating the Nrf2 pathway ${ }^{[14]}$. In this study, the expression levels of Nrf2, HO-1 and NQO1 in retinal tissues in normal group, low dose group and high dose group were higher than those in model group, consistent with the above research results. It can be inferred that NAR may relieve the inflammatory response and oxidative stress damage in DR through regulating the Nrf2/ARE signaling pathway, thereby exerting a protective effect on retinal cells.

In conclusion, NAR can effectively improve retinal cell apoptosis and oxidative damage in the treatment of DR, thereby protecting retinal ganglion cells. It may reduce the retinal damage caused by oxidative stress in DR rats through activating the Nrf2/ARE signaling pathway.

\section{Acknowledgments:}

This study was financially supported by Academic Fund of The First Hospital of Lanzhou University (No. ldyyyn2018-22).

\section{Conflict of interests:}

The authors declared no conflicts of interest. 


\section{REFERENCES}

1. Rondi S, Peddolla R, Venisetty RK. Neuro, cardio and reno protective activities of rosuvastatin in streptozotocin-induced type 2 diabetic rats undergoing treatment with metformin and glimepiride. J Adv Pharm Technol Res 2014;5(2):78-83.

2. de Melo IM, Ferreira CG, da Silva Souza EH, Almeida LL, de Sá FB, Neto CJ, et al. Melatonin regulates the expression of inflammatory cytokines, VEGF and apoptosis in diabetic retinopathy in rats. Chem Biol Interact 2020;327:109183.

3. Bellemo V, Lim ZW, Lim G, Nguyen QD, Xie Y, Yip MY, et al. Artificial intelligence using deep learning to screen for referable and vision-threatening diabetic retinopathy in Africa: a clinical validation study. Lancet Digit Health 2019;1(1):e3544.

4. Priscilla DH, Roy D, Suresh A, Kumar V, Thirumurugan K. Naringenin inhibits $\alpha$-glucosidase activity: a promising strategy for the regulation of postprandial hyperglycemia in high fat diet fed streptozotocin induced diabetic rats. Chem Biol Interact 2014;210:77-85.

5. Sykiotis GP, Bohmann D. Stress-activated Cap'n'collar transcription factors in aging and human disease. Sci Signal 2010;3(112):3-25.

6. Al-Dosari DI, Ahmed MM, Al-Rejaie SS, Alhomida AS, Ola MS. Flavonoid naringenin attenuates oxidative stress, apoptosis and improves neurotrophic effects in the diabetic rat retina. Nutrients 2017;9(10):1161.

7. Wang $K$, Chen Z, Huang L, Meng B, Zhou X, Wen X, et al. Naringenin reduces oxidative stress and improves mitochondrial dysfunction via activation of the Nrf2/ARE signaling pathway in neurons. Int J Mol Med 2017;40(5):158290.

8. Sandeep MS, Nandini CD. Influence of quercetin, naringenin and berberine on glucose transporters and insulin signalling molecules in brain of streptozotocin-induced diabetic rats. Biomed Pharmacother 2017;94:605-611.

9. Jia G, DeMarco VG, Sowers JR. Insulin resistance and hyperinsulinaemia in diabetic cardiomyopathy. Nat Rev Endocrinol 2016;12(3):144-53.

10. Chen MF, Liou SS, Kao ST, Liu IM. Erianin protects against high glucose-induced oxidative injury in renal tubular epithelial cells. Food Chem Toxicol 2019;126:97-105.

11. Gelen V, Şengül E. Antioxidant, anti-inflammatory and antiapoptotic effects of Naringin on cardiac damage induced by cisplatin. Indian J Tradit Know 2020;19:459-465.

12. Taguchi K, Motohashi H, Yamamoto M. Molecular mechanisms of the Keap1-Nrf2 pathway in stress response and cancer evolution. Genes Cells 2011;16(2):123-40.

13. Huang XX, RJ Su, Liu H. Inhibition of Artemisinin on oxidative stress injury induced by hydrogen peroxide in human retinal pigment epithelium cells. Recent Adv Ophthalmol 2019;39:12-17.

14. Sun Y, Xiu C, Liu W, Tao Y, Wang J, Qu YI. Grape seed proanthocyanidin extract protects the retina against early diabetic injury by activating the Nrf2 pathway. Exp Ther Med 2016;11:1253-8. 